\title{
The Presence of Intra- or Subretinal Fluid during the Loading Phase in the Treatment of Exudative Age-Related Macular Degeneration with Intravitreal Ranibizumab Assessed by Optical Coherence Tomography
}

\author{
Andreas Ebneter $^{\mathrm{a}}$ Boris Gekkiev ${ }^{\mathrm{a}}$ Bhuvan Chanana ${ }^{\mathrm{a}, \mathrm{b}}$ Sebastian Wolf ${ }^{\mathrm{a}}$ \\ Martin S. Zinkernage ${ }^{\mathrm{a}}$ \\ a Department of Ophthalmology, Inselspital, University of Bern, Bern, Switzerland; ${ }^{\text {b}}$ Department of Ophthalmology, \\ University College of Medical Sciences, Delhi, India
}

\section{Key Words}

Exudative age-related macular degeneration · Anti-vascular endothelial growth factor $\cdot$ Spectral-domain optical

coherence tomography $\cdot$ Retinal fluid $\cdot$ Scan protocol

\begin{abstract}
Purpose: To assess intra- and subretinal fluid during the loading phase with intravitreal ranibizumab in exudative age-related macular degeneration and to quantify the accuracy of crosshair scan spectral-domain optical coherence tomography with regard to retinal fluid. Methods: This is a retrospective study of 31 treatment-naive patients who received 3 monthly intravitreal ranibizumab injections. Visual acuity and the presence of retinal fluid were assessed at each visit using volume and crosshair scan protocols. Results: Visual acuity improved and central retinal thickness decreased significantly during the loading phase. However, retinal fluid persisted in two thirds of the patients. The accuracy of the crosshair scan to detect fluid was $93 \%$. Conclusions: A substantial proportion of eyes had persistent fluid after 3 months of ranibizumab injections. However, visual improve-
\end{abstract}

(c) 2015 S. Karger AG, Basel

0030-3755/15/0000-0000\$39.50/0 ment was independent of residual fluid. Message: Crosshair scans detect relevant collections of retinal fluid accurately and may be sufficient in daily clinical practice.

(c) 2015 S. Karger AG, Basel

\section{Introduction}

Age-related macular degeneration (AMD) is a chronic and degenerative disease and represents the leading cause of legal blindness in white persons [1]. The exudative (or wet) form presents with intra- (IRF) or subretinal fluid (SRF) because abnormal choroidal neovascularization leads to leakage of fluid into and underneath the neurosensory retina. Current treatment options for the neovascular component consist of anti-angiogenic substances that are able to reduce intra- and subretinal leakage and edema [2-4]. Anti-vascular endothelial growth factor antibodies such as ranibizumab (Lucentis; Genentech, Inc., South San Francisco, Calif., USA) have been shown to restore macular architecture to a remarkable degree, which often translates to a significant improvement of

\section{KARGER 125}

E-Mail karger@karger.com

www.karger.com/oph
Andreas Ebneter, MD, PhD

Universitätsklinik für Augenheilkunde

Inselspital

$\mathrm{CH}-3010$ Bern (Switzerland)

E-Mail ebneter.andreas@gmail.com 
visual acuity [5]. The initial treatment phase, the so-called loading phase, consisting of 3 monthly intravitreal injections of ranibizumab $0.5 \mathrm{mg} / 0.05 \mathrm{ml}$, has been shown to produce the major proportion of improvement in visual acuity and the main reduction of central retinal thickness (CRT) when assessed by optical coherence tomography (OCT) [6]. Whereas most clinical trials assessed CRT as parameter for treatment efficacy, the presence of IRF and/or SRF in OCT imaging is more indicative of exudative activity in clinical practice and is a decisive factor for recommending continuation of treatment for exudative AMD.

Using spectral-domain OCT (SD-OCT), discrete structures such as the photoreceptor inner/outer segments or the retinal pigment epithelium can be visualized. The high resolution allows the detection of minimal morphological changes occurring in the course of AMD. Scans can be obtained either from a single vertical and horizontal line, or as a crosshair scan combining a vertical and a horizontal line scan intersecting at a point of interest. Furthermore, owing to the short acquisition time of spectral-domain imaging, volume scans can be obtained from the central retina with high spatial resolution.

The aim of this study was to assess the presence of IRF or SRF during the loading phase in patients with exudative AMD receiving intravitreal anti-vascular endothelial growth factor therapy, using SD-OCT. Furthermore, the accuracy of crosshair SD-OCT imaging to detect SRF or IRF was assessed against high-resolution volume scanning as the gold standard.

\section{Methods}

In this retrospective interventional case series, 31 consecutive patients receiving initial treatment with ranibizumab for exudative AMD were identified from the procedure logs at the Retinal Service of the Department of Ophthalmology at the University Hospital Bern, Switzerland. All patients had clinically confirmed exudative AMD and had SD-OCT imaging performed before receiving treatment. Only treatment-naive patients were included in this study. The loading protocol was identical for all patients and consisted of 3 monthly intravitreal injections of ranibizumab 0.5 $\mathrm{mg} / 0.05 \mathrm{ml}$, as previously reported [7].

In addition to monthly SD-OCT imaging, best-corrected visual acuity (BCVA) was assessed monthly according to the ETDRS protocol with $\log$ MAR charts at 4 and $1 \mathrm{~m}$, respectively. Spectralis SD-OCT (Heidelberg Engineering, Heidelberg, Germany, software version 5.3) scans were acquired using an established protocol consisting of both a crosshair and a volume scan. The crosshair scan consisted of a vertical and a horizontal scan line intersecting at the fovea. The volume scan, covering $20^{\circ} \times 20^{\circ}$, comprised 49 parallel B-scans separated by $121 \mu \mathrm{m}$, whereby each B-scan was the average of 9 frames (automated real-time repetition rate $=9$ ) consisting of 512 A-scans.

Each scan was reviewed by 2 independent assessors (A.E., M.S.Z.) for the presence of IRF or SRF. The presence of IRF, SRF or macular fluid (MF), a combination of SRF and IRF, was recorded for each monthly follow-up visit during the loading phase. For each type of fluid collection, subgroup analysis of CRT and BCVA was performed. CRT was determined automatically by the software as the distance from the internal limiting membrane of the retina to the outer border of the retinal pigment epithelium, excluding any sub-retinal pigment epithelium structures like choroidal neovascularization membranes.

Ethics approval (KEK No. 093/13) to conduct this study was obtained from the local Ethics Committee, which is working in accordance with the ICH-GCP guidelines. The need for written consent from each individual patient was waived because of the retrospective nature of the study. The data were analyzed with paired $t$ test and one-way ANOVA as appropriate. $\mathrm{p}<0.05$ was considered statistically significant. Error bars in the graphs represent standard errors of the mean (SEM). Statistical analysis was performed using the Prism GraphPad ${ }^{\circledR}$ software package (Prism 5; GraphPad Software Inc., La Jolla, Calif., USA).

\section{Results}

Thirty-one patients, seen between 2010 and 2012 at the Retinal Service of the Department of Ophthalmology at the University Hospital Bern, Switzerland, had complete data for 3 consecutive months and were evaluated in this study. The mean age of the patients at the time of diagnosis of exudative AMD was 77 years (range: 57-93). The mean BCVA at baseline was 50.9 letters (range: 5-86) with a CRT of $491 \mu \mathrm{m}$ (range: 265-808). BCVA increased from a mean \pm SD of $50.9 \pm 16.4$ letters at baseline to 57.5 \pm 12.8 at month $3(\mathrm{p}=0.003)$. CRT was $491 \pm 138 \mu \mathrm{m}$ at baseline and $374 \pm 145 \mu \mathrm{m}$ at month $3(\mathrm{p}<0.0001)$. The changes in BCVA and CRT in our patient cohort during the loading phase with ranibizumab were similar to other published data [8] and are summarized in figure 1.

The percentage of eyes with any fluid 1 month after the third injection was $71 \%$ when assessed by volume scan and $61 \%$ with crosshair scan. The subgroup analysis showed that eyes with IRF (33\%) or MF (31\%) seemed to have a higher chance of complete resolution of fluid after 3 intravitreal injections compared to eyes with SRF (22\%). There was rapid improvement of BCVA in the MF group during the loading phase, from $46.4 \pm 18.4$ letters at baseline to $54.0 \pm 14.1$ at month 3 (fig. $2 \mathrm{a}$ ), whereas SRF patients displayed a slower visual recovery, but starting from better visual acuity, from $59.4 \pm 13.3$ letters at baseline to $65.2 \pm 9.1$ at month 3 (fig. 2 b). In the IRF group, the visual gain was 5.3 letters, from $50.0 \pm 10.8$ letters at 
Fig. 1. Mean changes from baseline in visual acuity and CRT during the loading phase. a Mean gain in ETDRS letters from baseline visual acuity at 1 through 3 months after treatment start. At each time point, the increase is significant compared to baseline BCVA: 4.3 letters at month $1(\mathrm{p}=$ $0.012), 5.8$ letters at month $2(\mathrm{p}=0.031)$ and 6.6 letters at month $3(\mathrm{p}=0.003)$. b Decrease in CRT compared to baseline. The decrease was $92 \mu \mathrm{m}$ at month $1(\mathrm{p}=0.0008)$, $133 \mu \mathrm{m}$ at month $2(\mathrm{p}<0.0001)$ and $117 \mu \mathrm{m}$ at month $3(\mathrm{p}<0.0001)$.

Fig. 2. Subgroup analysis of BCVA. BCVA is illustrated at baseline and 3 months after the first intravitreal injection. Subgroups were defined as eyes with both IRF and SRF (i.e. MF, a), SRF only (b), and IRF only (c). Baseline BCVA did not seem to differ between the subgroups (one-way ANOVA, $\mathrm{p}=0.160$ ), nor did CRT (one-way ANOVA, $\mathrm{p}=0.134)$. Letter gain and decrease in CRT were not statistically different between the subgroups (one-way ANOVA, p $=0.893$ and $\mathrm{p}=0.308$, respectively).
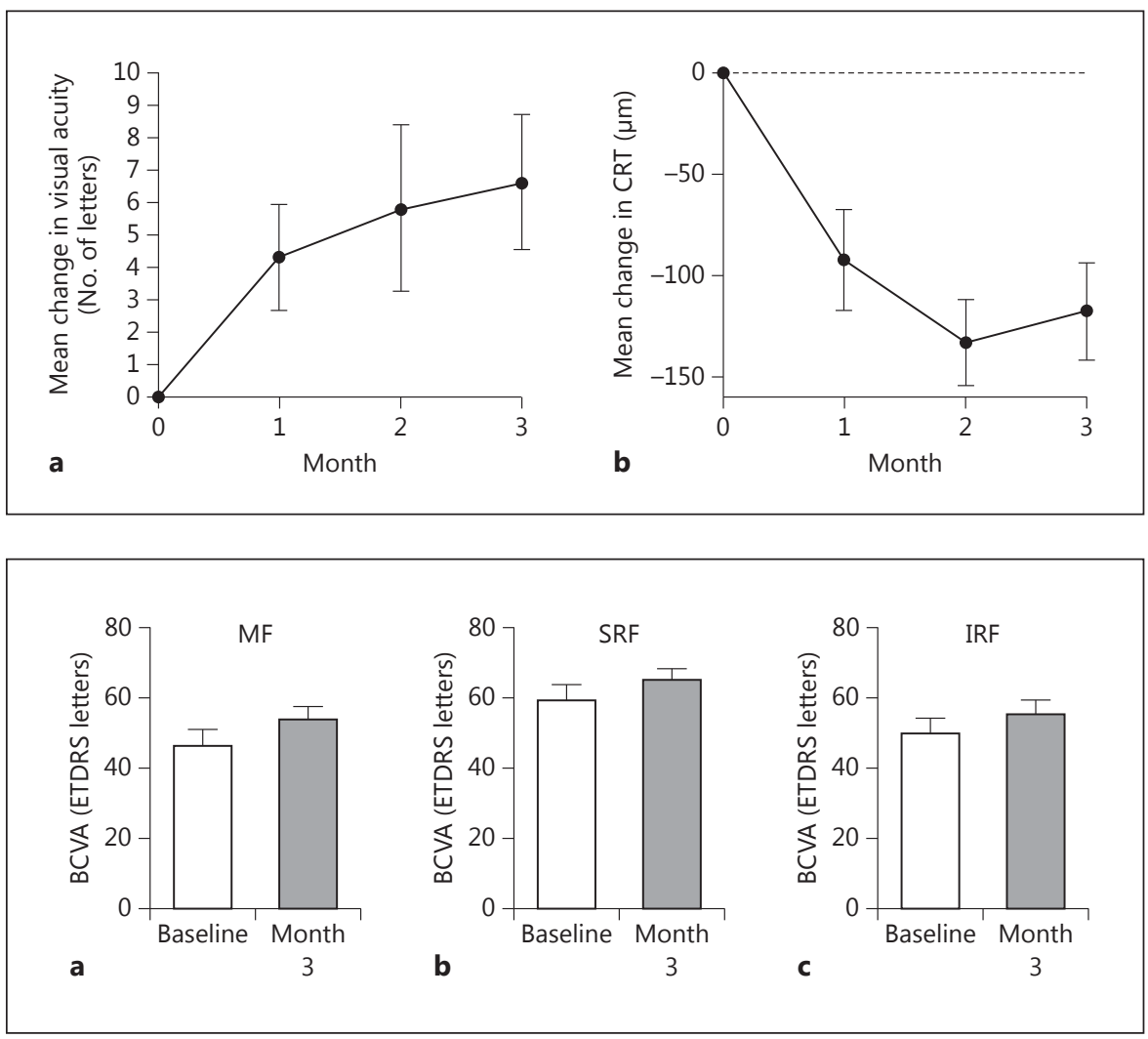

baseline to $55.3 \pm 10.0$ at month 3 (fig. 2c). Neither baseline nor improvement of BCVA was statistically significant between the groups (one-way ANOVA).

The occurrence of pigment epithelium detachments (PED) was also investigated. In 17 eyes, there was a PED present at the onset of the treatment, which persisted throughout the loading phase. In 2 patients, a PED was present at the beginning of the observation period and disappeared in the course of the loading phase (fig. 3a-c). One patient developed a persisting PED during the loading treatment, whereas 10 eyes never showed evidence of a PED. In 1 eye, a PED appeared after the first intravitreal injection of ranibizumab but then subsequently dissolved after another injection (fig. 3d-f).

Eyes without IRF or SRF at the conclusion of the loading phase had a significantly larger decrease in CRT when compared to eyes with persistent IRF or SRF $(p=0.002)$. Whereas in the former group, CRT diminished by $226 \pm$ $159 \mu \mathrm{m}$, in the latter, it decreased by only $73 \pm 94 \mu \mathrm{m}$. However, there was no difference in the change of BCVA between these 2 groups ( $\mathrm{p}=0.927)$, suggesting that persisting fluid does not significantly affect visual acuity (fig. 4) early during treatment with ranibizumab.

Intraretinal Fluid during Loading Phase
The overall sensitivity of crosshair scans to detect IRF or SRF when benchmarked against volume scans was 93\% $(\mathrm{n}=122)$. Crosshair scans were more sensitive to detect SRF (93\%) than IRF (69\%). A representative case in which IRF was missed on the crosshair scan is illustrated in figure 5.

\section{Discussion}

In our study, we found a high percentage of eyes displaying persisting IRF or SRF after initiation of treatment with ranibizumab. To our knowledge, this is the first report showing the outcome after the initial loading phase with ranibizumab with regard to IRF or SRF. After 3 injections, $71 \%$ of the eyes still displayed IRF or SRF. After 1 injection of ranibizumab, $80 \%$ of the eyes had IRF or SRF, which is in keeping with recently reported data from the CATT study. In the CATT study, at 4 weeks, 161 of $586(27.5 \%)$ patients treated with ranibizumab had no fluid on OCT, and after 1 year, only $24 \%$ had no IRF or SRF on OCT in the ranibizumab treatment-as-needed group [9]. 


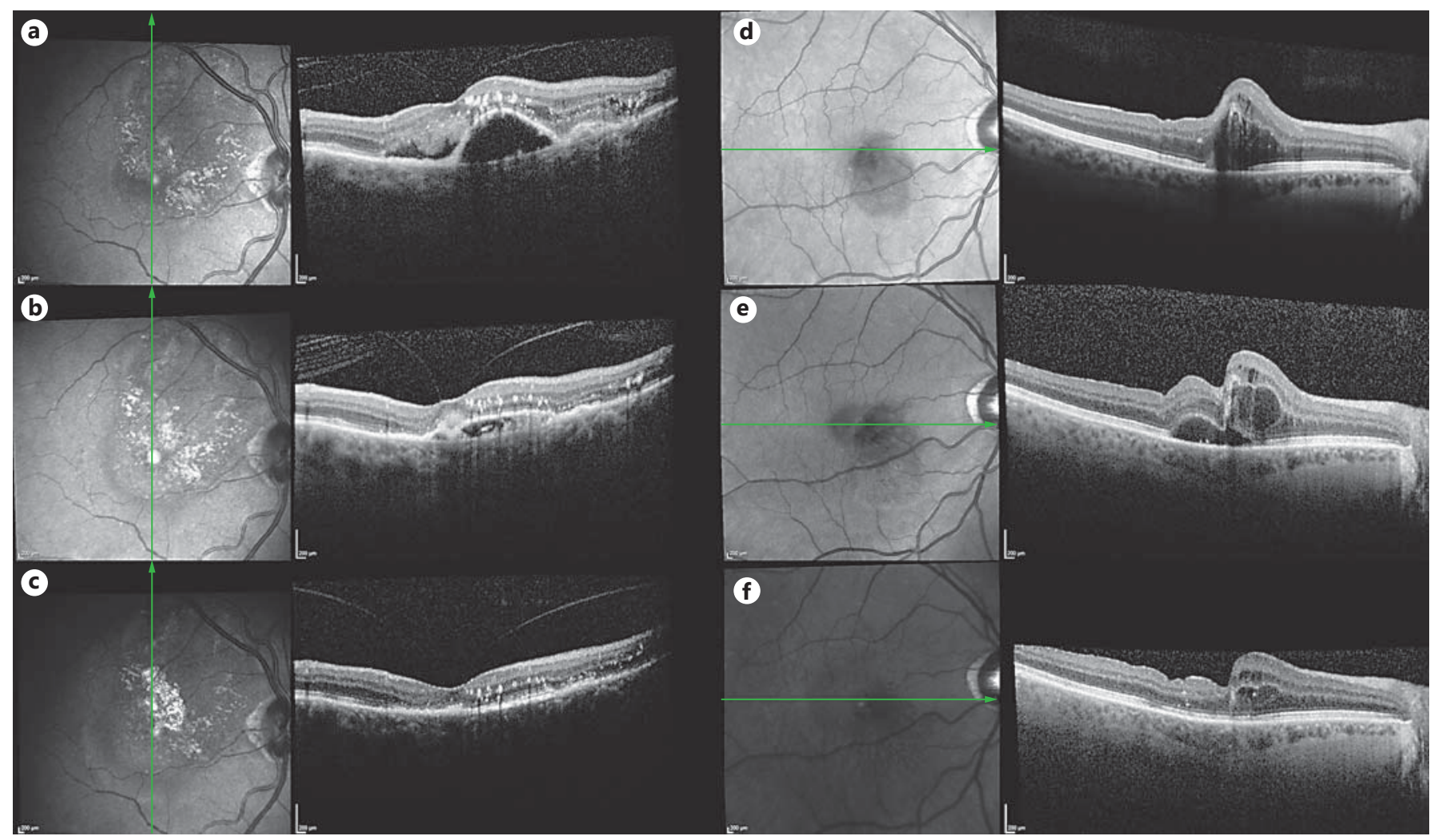

Fig. 3. Case histories representing different comportments of PED during the loading phase with ranibizumab. In case 1 (a-c), the PED present at baseline (a) gradually vanishes and is not detectable at the month 2 visit (c) any more. In the second case (d-f), a PED, not detectable at baseline (d), appears at the month 1 visit (e), but is no longer present a month later (f).

Fig. 4. Subgroup analysis of BCVA and CRT based on the presence or absence of fluid at conclusion of the loading phase. Both subgroups had a similar increase in visual acuity (a). Interestingly, the quick return of CRT to the normal range (b) was not paralleled by a swifter visual recovery.

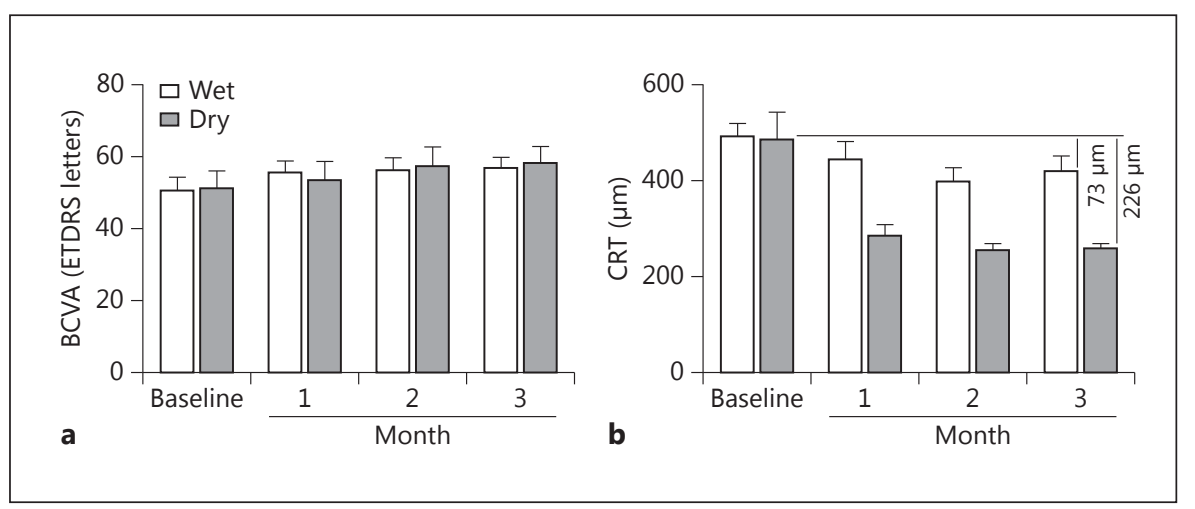

Several salient OCT features during the loading phase of exudative AMD merit further discussion. Our data suggest that after the loading phase, only $29 \%$ of the patients display no SRF or IRF and that therefore, $71 \%$ of the patients will require further monthly injections. Whereas $20 \%$ of the patients were completely dry after the first injection, the proportion of patients displaying no IRF or SRF after the second or third injection increased only marginally to $29 \%$ after the third injection. Interestingly, the presence or absence of fluid did not affect the visual recovery in the short loading phase, although the persistence of fluid in the long term can result in bad out- 


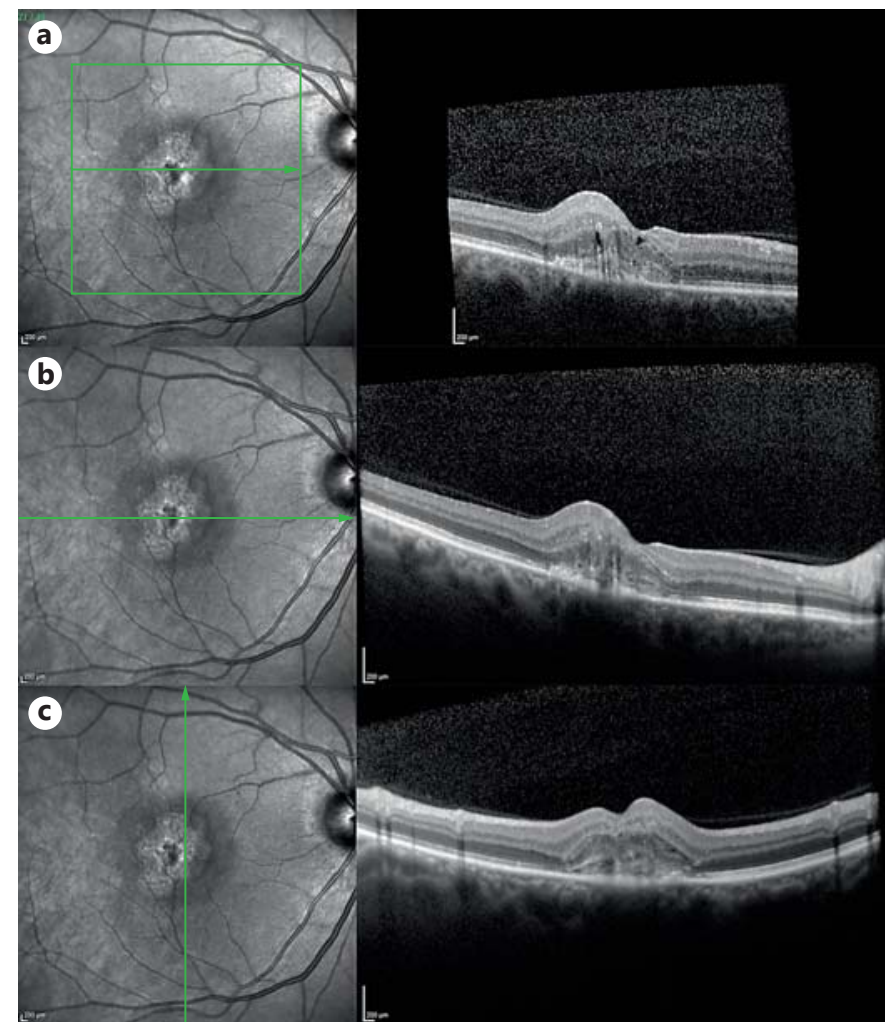

Fig. 5. Representative case history illustrating the somewhat lower sensitivity of a crosshair scan to detect small amounts of IRF. b, c OCT scan along the green line. No IRF is visible. However, in the volume scan, on a horizontal scan taken slightly above the scan in b, IRF is clearly present (a).

comes [10]. There seems to be a large degree of variability in the accumulation of IRF or SRF as the percentage of patients having been dry at any stage during the loading phase was $50 \%$ in the MF group, $23 \%$ in the SRF group and $33 \%$ in the IRF group.

The decrease of CRT after the loading phase was significantly larger in patients with no residual fluid at the end of the loading phase when compared to patients with persisting IRF or SRF. In all large studies investigating the effect of ranibizumab, 3 consecutive monthly injections appeared optimal and the majority of patients experienced the most gain of visual acuity in this phase of treatment [11]. During the loading phase, the largest gain in visual acuity occurred after the first injection. This is in keeping with our data. However, persisting IRF or SRF did not influence the improvement in BCVA during the loading phase with ranibizumab in our study. Even 6 months after the first injection, patients with persisting

Intraretinal Fluid during Loading Phase
IRF or SRF did not have significantly worse visual acuity when compared to the group that was completely dry (data not shown). In the subgroup analysis, the location of the fluid neither influenced baseline visual acuity nor the gain in visual acuity.

Information about the changes in different macular compartments with regard to treatment response to ranibizumab is essential to identify parameters to guide treatment and retreatment criteria. In this study, we showed that SD-OCT provides an excellent tool with high resolution to identify the dynamics of IRF or SRF during the loading phase with ranibizumab in the treatment of exudative AMD. However, single crosshair OCT scans may fail to detect some cases with parafoveal exudative activity. Interestingly, this is particularly true after the first injection, possibly due to compartmentalization of fluid and the establishment of residual parafoveal cystoid spaces.

In conclusion, analysis of OCT images during the loading phase with ranibizumab showed that the majority of patients had persisting IRF or SRF. The presence of persisting IRF or SRF during treatment with ranibizumab is increasingly apparent due to the ability of SD-OCT to swiftly acquire high-resolution volume scans and detect even smaller fluid-filled spaces. We showed that persisting fluid during the induction phase did not lead to a slower gain in BCVA when compared to eyes that were dry at the end of the loading phase.

\section{Disclosure Statement}

A.E.: lecture fees from Bayer AG; B.G.: none; B.C.: none; S.W.: consultant/advisor at and financial support from Heidelberg Engineering, consultant/advisor at Zeiss, consultant/advisor at Novartis AG, consultant/advisor at Bayer AG, and consultant/advisor at and financial support from Optos plc; M.S.Z.: financial support from Heidelberg Engineering, consultant/advisor at Novartis AG, and consultant/advisor at Bayer AG.

References

1 Congdon N, O’Colmain B, Klaver CC, Klein R, Munoz B, Friedman DS, Kempen J, Taylor HR, Mitchell P: Causes and prevalence of visual impairment among adults in the United States. Arch Ophthalmol 2004;122: 477-485.

2 Cruess AF, Berger A, Colleaux K, Greve M, Harvey P, Kertes PJ, Sheidow T, Tourville E, Williams G, Wong D: Canadian expert consensus: optimal treatment of neovascular agerelated macular degeneration. Can J Ophthalmol 2012;47:227-235. 
3 Koh A, Lim TH, Au Eong KG, Chee C, Ong SG, Tan N, Yeo I, Wong D: Optimising the management of choroidal neovascularisation in Asian patients: consensus on treatment recommendations for anti-VEGF therapy. Singapore Med J 2011;52:232-240.

4 Lally DR, Gerstenblith AT, Regillo CD: Preferred therapies for neovascular age-related macular degeneration. Curr Opin Ophthalmol 2012;23:182-188.

5 Mitchell P, Korobelnik JF, Lanzetta P, Holz FG, Prunte C, Schmidt-Erfurth U, Tano Y, Wolf S: Ranibizumab (Lucentis) in neovascular age-related macular degeneration: evidence from clinical trials. Br J Ophthalmol 2010;94:2-13.
6 Fung AE, Lalwani GA, Rosenfeld PJ, Dubovy SR, Michels S, Feuer WJ, Puliafito CA, Davis JL, Flynn HW Jr, Esquiabro M: An optical coherence tomography-guided, variable dosing regimen with intravitreal ranibizumab ( $\mathrm{Lu}-$ centis) for neovascular age-related macular degeneration. Am J Ophthalmol 2007;143: 566-583.

7 Veritti D, Sarao V, Lanzetta P: Neovascular age-related macular degeneration. Ophthalmologica 2012;227(suppl 1):11-20.

8 Rosenfeld PJ, Brown DM, Heier JS, Boyer DS, Kaiser PK, Chung CY, Kim RY: Ranibizumab for neovascular age-related macular degeneration. N Engl J Med 2006;355:1419-1431.
9 Martin DF, Maguire MG, Ying GS, Grunwald JE, Fine SL, Jaffe GJ: Ranibizumab and bevacizumab for neovascular age-related macular degeneration. N Engl J Med 2011;364:18971908.

10 Rothenbuehler SP, Waeber D, Brinkmann CK, Wolf S, Wolf-Schnurrbusch UE: Effects of ranibizumab in patients with subfoveal choroidal neovascularization attributable to age-related macular degeneration. Am J Ophthalmol 2009; 147:831-837.

11 Brown DM, Kaiser PK, Michels M, Soubrane G, Heier JS, Kim RY, Sy JP, Schneider S: Ranibizumab versus verteporfin for neovascular age-related macular degeneration. N Engl J Med 2006;355:1432-1444. 